\title{
Nondysraphic Intradural Spinal Lipoma: A Rare Case in a Child
}

\author{
Antonio GL J r. ${ }^{1 *}$, Abreu NMP1, Leal MVB', \\ Rodrigues J PC ${ }^{1}$, Malveira $\mathrm{C}^{1}$, Gomes $\mathrm{LVM}^{2}$ and \\ Coimbra PPA ${ }^{1}$ \\ ${ }^{1}$ Radiology Unit, Hospital Antonio Prudente, Fortaleza, \\ CE, Brazil \\ ${ }^{2}$ UniRV, Goianésia, GO, Brazil \\ *Corresponding author: Antonio Gomes Lima J unior, \\ Radiology Unit, Hospital Antonio Prudente, Fortaleza, \\ CE, Brazil
}

Received: July 20, 2021; Accepted: August 24, 2021; Published: August 31, 2021

\section{Introduction}

Spinal cord lipomas represent a group of rare and benign tumors. It accounts for about $1 \%$ of all intramedullary tumors. These are injuries associated with spinal dysraphisms. The absence of association with dysraphism makes the lesion even rarer, representing about $1 \%$ of cases of spinal cord lipomas. The case to be discussed is a child diagnosed with intradural lipoma without associated dysraphism.

\section{Case Presentation}

The case reported is of an 8-month-old child, who started a condition of excessive crying associated with stooped posture in the cervical and thoracic region at 3 months of age, in a progressive manner. Report evaluated by an orthopedist who discarded pathological processes. She had motor development delay, with difficulty in supporting her neck, trunk, without sitting down at 7 months. Hospitalized at 8 months due to gastrointestinal symptoms, where fontanelle bulging was observed, and a blood CT was requested, which showed an expansive lesion in the posterior fossa with insinuation to the spinal canal. Blood MRI was performed showing an image compatible with spinal cord lipoma, without signs of associated dysraphism. She underwent a surgical procedure, where the tumor was partially resected due to the extension of the lesion. $\mathrm{He}$ is still hospitalized in ventricle bypass planning (Figure 1-4).

\section{Discussion}

Medullary lipomas are very rare entities, especially when there is no association with dysraphisms, representing about $1 \%$ of all spinal lipomas and 5-7\% in children, as reported in this case [1]. Most are intradural (60\%), while the rest are extradural (40\%). The clinical presentation and its neurological component are quite variable depending on the size and location of the lesion, but it usually evolves insidiously [2]. The case in question presented early symptoms with motor delay due to the significant tumor volume [3]. This condition is more commonly located in higher regions of the spinal cord, especially in the projections of the $4^{\text {th }}$ and $5^{\text {th }}$ thoracic vertebrae. In the case reported here, there is involvement from the $4^{\text {th }}$ vertebra to the posterior cranial fossa. MRI is the main diagnostic method, not only for confirmation, but also for evaluating the involvement of adjacent

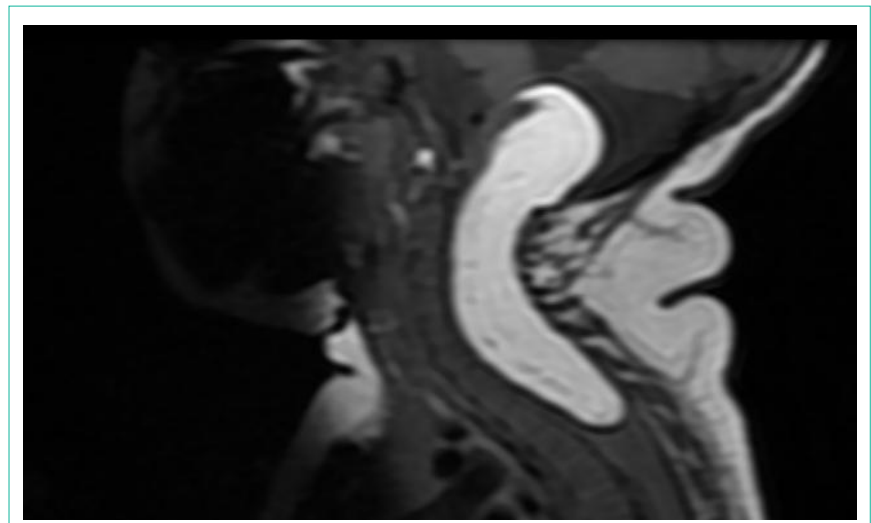

Figure 1: Sagittal/T1 image - T1-weighted hyperintense lesion extending from the craniocervical transition to the thoracic spine.

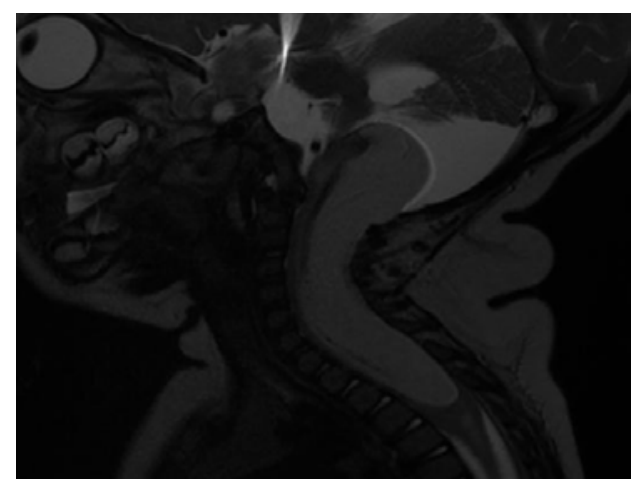

Figure 2: Sagittal image/T2 - Lesion with marked drop in T2-weighted signal extending from the craniocervical transition to the D4 thoracic vertebra.

structures. They usually have a high signal on T1 and a low signal on T2. The treatment of these injuries is surgical, with the main objective of spinal cord decompression, without the need for complete excision of the tumor, with several reports of worse postoperative results in case of total excision. Contraindicated in asymptomatic patients. In the case patient, a partial excision with spinal decompression was performed [4].
Austin J Radiol - Volume 8 Issue 8 - 2021

ISSN : 2473-0637 | www.austinpublishing group.com

Antonio et al. () All rights are reserved
Citation: Antonio GL Jr, Abreu NMP, Leal MVB, Rodrigues JPC, Malveira C, Gomes LVM, et al. Nondysraphic Intradural Spinal Lipoma: A Rare Case in a Child. Austin J Radiol. 2021; 8(8): 1157. 


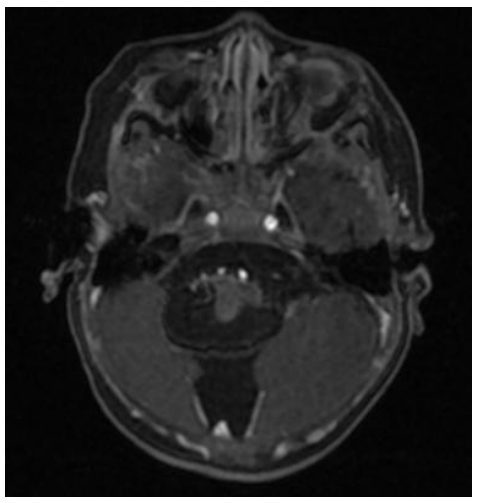

Figure 3: Axial/T1 Fat-Sat Pos Contrast image - Lesion with marked signal drop in the sequences with fat saturation and no contrast uptake, intradural and difficult to characterize if extra or intramedullary.

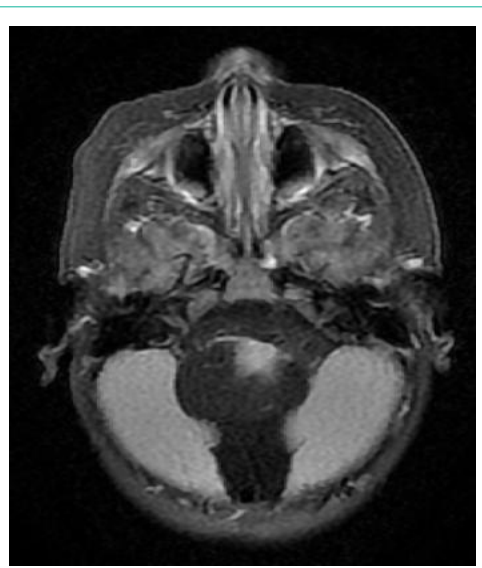

Figure 4: Axial image/FLAIR - Medulla undergoes compressive effect of the lesion with change in signal/hypersignal in the FLAIR sequence, translating compressive myelopathy.

Lobulated expansive formation, well delimited and with regular contours, with signal intensity similar to fat, is characterized in the medullary canal, extending from a thoracocervical transition at the level of the vertebral body of D4 to the posterior cranial fossa, at the level of the obex, suggestive of lesion of lipomatous origin, measuring at least $8.9 \times 4.0 \times 3.1 \mathrm{~cm}$ in its longest axes (caudal blood/lateral/ anteroposterior) [5].
The lesion has an intradural situation, difficult to characterize whether intra or extramedullary, but occupying the posterior aspect of the spinal canal, determining its enlargement, with anterolateral displacement to the left of the spinal cord and bulbomedullary transition, which is compressed and deformed, areas of thinning and signal alteration characterized by hypersignal on T2/STIR, denoting some degree of compressive myelopathy.

\section{Conclusion}

Intradural lipomas without association with dysraphism are rare conditions common in the literature, where the diagnosis is usually made clearly and objectively with MRI. Surgical treatment has as main objective the decompression of the spinal canal, with no need for complete excision of the lesion and contraindicated in asymptomatic patients.

Although there has been improvement in prenatal diagnosis in recent years, adequate clinical, pre- and post-surgical management is still quite challenging, mainly due to the rarity of the disease, generating limited information for the production of adequate treatment protocols of this pathological condition.

\section{References}

1. So Wan-Soo, Lee Woo-Jong, Choi Ha-Young, Eun Jong-Pil. Spinal intramedullary lipoma without dysraphism. Journal of Korean Neurosurgical Society. 2007; 42: 42-45.

2. Pasalic I, Brgic K, Nemir J, Kolenc D, Njiric N \& Mrak G. Intramedullary Spinal Cord Lipoma Mimicking a Late Subacute Hematoma. Asian journal of neurosurgery. 2018; 13: 1282-1284.

3. Falavigna, Asdrubal, Segatto, Ana Claudia, \& Salgado, Karine. A rare case of intramedullary lipoma associated with cyst. Arquivos de Neuro-Psiquiatria. 2001; 59: 112-115.

4. Vasudha Patwardhan, Tufail Patanakar, Diane Armao and Suresh $\mathrm{K}$ Mukherji. MR Imaging Findings of Intramedullary Lipomas. American Journal of Roentgenology. 2000; 174: 1792-1793.

5. Bekar Ahmet, Sahin Soner, Taşkapiloğlu Ö, Aksoy Kiymet, Tolunay Ş. Intradural spinal lipoma: Report of a thoracic case and a lumbar case. Turkish Neurosurgery. 2004; 14: 52-56. 